\title{
Impact of particle shape on the morphology of noctilucent clouds
}

\author{
J. Kiliani ${ }^{1, \text { a }}$, G. Baumgarten ${ }^{1}$, F.-J. Lübken ${ }^{1}$, and U. Berger ${ }^{1}$ \\ ${ }^{1}$ Leibniz Institute of Atmospheric Physics at Rostock University, Schlossstraße 6, 18225 Kühlungsborn, Germany \\ a now at: Max-Planck Institute for Meteorology, Bundesstraße 53, 20146 Hamburg, Germany
}

Correspondence to: G. Baumgarten (baumgarten@iap-kborn.de)

Received: 27 April 2015 - Published in Atmos. Chem. Phys. Discuss.: 15 June 2015

Revised: 9 October 2015 - Accepted: 25 October 2015 - Published: 19 November 2015

\begin{abstract}
Noctilucent clouds (NLCs) occur during summer in the polar region at altitudes around $83 \mathrm{~km}$. They consist of ice particles with a typical size around $50 \mathrm{~nm}$. The shape of NLC particles is less well known but is important both for interpreting optical measurements and modeling ice cloud characteristics. In this paper, NLC modeling of microphysics and optics is adapted to use cylindrical instead of spherical particle shape. The optical properties of the resulting ice clouds are compared directly to NLC three-color measurements by the Arctic Lidar Observatory for Middle Atmosphere Research (ALOMAR) Rayleigh/Mie/Raman (RMR) lidar between 1998 and 2014. Shape distributions including both needle- and disc-shaped particles are consistent with lidar measurements. The best agreement occurs if disc shapes are $60 \%$ more common than needles, with a mean axis ratio of 2.8. Cylindrical particles cause stronger ice clouds on average than spherical shapes with an increase of backscatter at $532 \mathrm{~nm}$ by $\approx 30 \%$ and about $20 \%$ in ice mass density. This difference is less pronounced for bright than for weak ice clouds. Cylindrical shapes also cause NLCs to have larger but a smaller number of ice particles than for spherical shapes.
\end{abstract}

\section{Introduction}

Noctilucent clouds (NLCs), also called polar mesospheric clouds (PMCs), occur in the polar region at altitudes around $83 \mathrm{~km}$. NLCs only form during summer, when the upper mesosphere is coldest (below $130 \mathrm{~K}$ ) and the amount of water vapor is enhanced due to transport by atmospheric circulation (Holton, 1983). NLCs consist of ice particles with $r \approx 50 \mathrm{~nm}$ which form by heterogeneous nucleation, for example around meteoric dust particles (Turco et al., 1982).
The size of mesospheric ice particles can be inferred with optical instruments such as lidar, which measure backscattered light at multiple wavelengths. Using microphysical modeling in this context requires simulating particle shape, since measurements indicate that NLC particles in general are not spherical.

For example Baumgarten et al. (2002) indicate needlelike particles with a diameter-over-length ratio $(\varepsilon)$ of less than 0.4. Eremenko et al. (2005) indicate needle- or plate-like particles with $\varepsilon \approx 0.5$ or $\varepsilon \approx 2$. Rapp et al. (2007) estimated $\varepsilon \approx 0.2$ or $\varepsilon \approx 7$. The most extensive data set is from the SOFIE (Solar Occultation For Ice Experiment) instrument, with mean $\varepsilon \approx 0.5$ or $\varepsilon \approx 2$ (Hervig et al., 2009a; Hervig and Gordley, 2010).

In this paper, the formation of noctilucent clouds consisting of non-spherical particles is studied in order to allow for a direct comparison of lidar measurements to model data. This also allows for predictions about the effects of particle shape on the formation of NLC layers.

\section{Analysis methods}

\subsection{Model description}

In this study, the size of noctilucent cloud particles is calculated using the Mesospheric Ice Microphysics And tranSport model (MIMAS), formerly named LIMA/ICE (Berger and Lübken, 2006; Lübken et al., 2009). MIMAS is a 3-D Lagrangian ice particle model for the polar mesosphere. Water vapor and an ensemble of 40 million condensation nuclei are transported by winds taken from an atmospheric circulation model, usually LIMA (Leibniz-Institute Middle Atmosphere model; Berger, 2008). When the ambient air is supersaturated, condensation nuclei are coated with ice. When they 
grow in particle size, these ice particles sediment to lower altitudes within a few hours, where they eventually sublimate. The current version of MIMAS is described in detail in Kiliani (2014). More details about earlier model versions using dynamics from COMMA/IAP are found in Berger and von Zahn (2002), von Zahn and Berger (2003), and Berger and von Zahn (2007), about MIMAS used with LIMA dynamics (LIMA/ICE) in Lübken et al. (2009, 2013) and Berger and Lübken (2015).

\subsection{Microphysics of non-spherical particles}

In LIMA/ICE all ice particles are assumed to be spherical. For most purposes, this simplification is reasonable and widely used in NLC models, for example in the Community Aerosol and Radiation Model for Atmospheres (CARMA) (Megner, 2011; Merkel et al., 2009; Bardeen et al., 2010). For this study, cylindrical particle shape is used in addition to spherical particles. Pruppacher and Klett (1997) found a prevalence of cubic ice at the temperature and pressure range of NLCs. More recent studies indicate a mixture of cubic and hexagonal ice (stacking disordered) (Murray et al., 2015). Direct measurements of tropospheric ice indicate strongly elongated or flattened shapes (Hobbs et al., 1974). To simplify the model representation of non-spherical shapes, we treat these as cylinders. We define a cylinder by its axis ratio $\varepsilon=\frac{d}{h}$ and volume-equivalent radius $r=$ $\sqrt[3]{\frac{3}{16} d^{2} \cdot h}$, with base diameter $d$ and length of symmetry axis $h$ (Fig. 1).

Each model ice particle thus has a specified shape, in addition to a spatial coordinate and radius. $\varepsilon$ is assumed to stay constant in time; i.e., the axis ratio does not change when cylindrical particles grow or sublimate (see Sect. 4). The particle shape modifies the growth rate $\frac{\mathrm{d} r}{\mathrm{~d} t}$; this effect can be approximated as

$\left.\frac{\mathrm{d} r}{\mathrm{~d} t}\right|_{\text {cylinder }}=\left.\Phi_{\mathrm{d} r \mathrm{~d} t} \cdot \frac{\mathrm{d} r}{\mathrm{~d} t}\right|_{\text {sphere }}$,

where the factor $\Phi_{\mathrm{d} r \mathrm{~d} t}$ depends on the particle shape, specifically the surface area $S$ (e.g., Turco et al., 1982):

$\Phi_{\mathrm{d} r \mathrm{~d} t}=\frac{S_{\text {cylinder }}(r, \varepsilon)}{S_{\text {sphere }}(r)}=\left(\frac{1}{\varepsilon}+\frac{1}{2}\right)\left(\frac{2 \varepsilon}{3}\right)^{\frac{2}{3}}$.

The mass change rate of ice particles is proportional to the frequency of collisions with surrounding air molecules, and thus to the surface area $S$. Since $S$ at a given volume is smallest for spheres, a non-spherical shape makes particles grow or sublimate faster. Equation (1) is best suited for larger particles: shape also modifies the Kelvin effect since that depends on the mean particle curvature, but this effect is only pronounced for particles smaller than $\approx 5 \mathrm{~nm}$ and is currently not included in our simulations.

Elongated and flattened ice particles also sediment more slowly than spherical ones. While the speed of sedimenta-

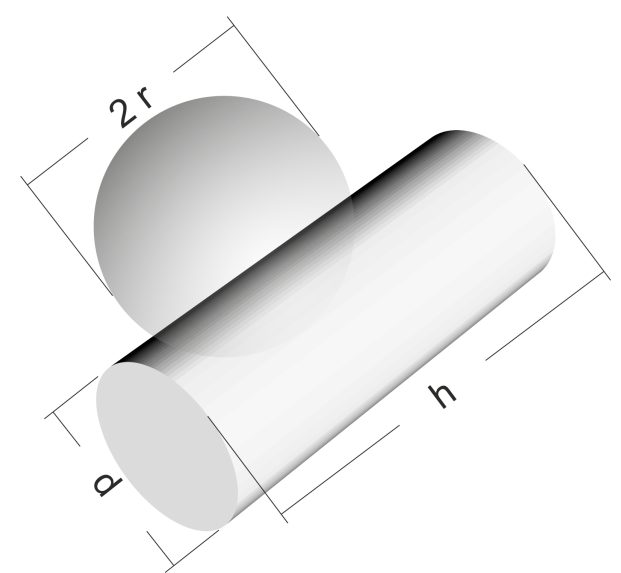

Figure 1. Nomenclature used for cylindrical ice particles.

tion depends on the particles' orientation, random orientation can be assumed for mesospheric ice: by way of their small size, rotational Brownian motion contributes a significant part of their thermal energy, equally distributed over all axes. Gadsden (1983) estimated this rotation at $\approx 10^{6} \mathrm{~Hz}$, so the randomization process is orders of magnitude faster than the MIMAS resolution of $90 \mathrm{~s}$. The random particle orientation allows calculating an average vertical cross section for collisions with air molecules, which determines the sedimentation rate $w_{\mathrm{s}}$. As this cross section increases for elongated and flattened particles for a given volume, needle- and discshaped particles fall more slowly than spheres:

$\Phi_{\text {sedi }}=\frac{w_{\mathrm{s}, \text { cylinder }}}{w_{\mathrm{s}, \text { sphere }}}=\frac{\left(\frac{4}{9 \varepsilon}\right)^{1 / 6} \cdot\left(1+\frac{\pi}{8}\right)}{\frac{1}{4} \sqrt{3+\frac{\pi}{2}+\varepsilon} \cdot \sqrt{2+\frac{2}{\varepsilon}+\frac{\pi}{2}}}$.

The correction factors $\Phi_{\mathrm{d} r \mathrm{~d} t}$ and $\Phi_{\text {sedi }}$ are shown in Fig. 2. $\Phi_{\mathrm{d} r \mathrm{~d} t}$ and $\Phi_{\text {sedi }}$ are around 2 and 0.5 , respectively, for flattened particles with $\varepsilon=10$. For elongated particles $(\varepsilon=$ 0.1 ), the values are closer to 1 than for $\varepsilon=10$, indicating that "disc"-shaped particles affect NLC microphysics more than "needle"-shaped ones. Using a smaller range of particle shapes from $\approx 0.3$ (needles) to 3 (discs) consistent with Hervig and Gordley (2010) leads to $\Phi_{\mathrm{d} r \mathrm{~d} t}<1.3$ and $\Phi_{\text {sedi }}>$ 0.7. Both correction factors differ from 1 for $\varepsilon=1$ : cylindrical particles with equal diameter and height grow faster and fall more slowly than spherical particles. Also shown in Fig. 2, $\left.\frac{\mathrm{d} r}{\mathrm{~d} t}\right|_{\text {spheroid }}<\left.\frac{\mathrm{d} r}{\mathrm{~d} t}\right|_{\text {cylinder }}$ for $\varepsilon$ values close to 1 . Only oblate spheroids with $\varepsilon>4$ grow faster than flattened cylinders at the same axis ratio.

Finally, optical particle properties are also affected by particle shape: in the standard version of MIMAS, backscatter coefficients are calculated only for green light at $532 \mathrm{~nm}$, assuming spherical shape. For this paper this is expanded to include randomly oriented cylindrical particles with variable axis ratios, calculated with the T-matrix method (Mishchenko and Travis, 1998) at 355, 532 and $1064 \mathrm{~nm}$. 


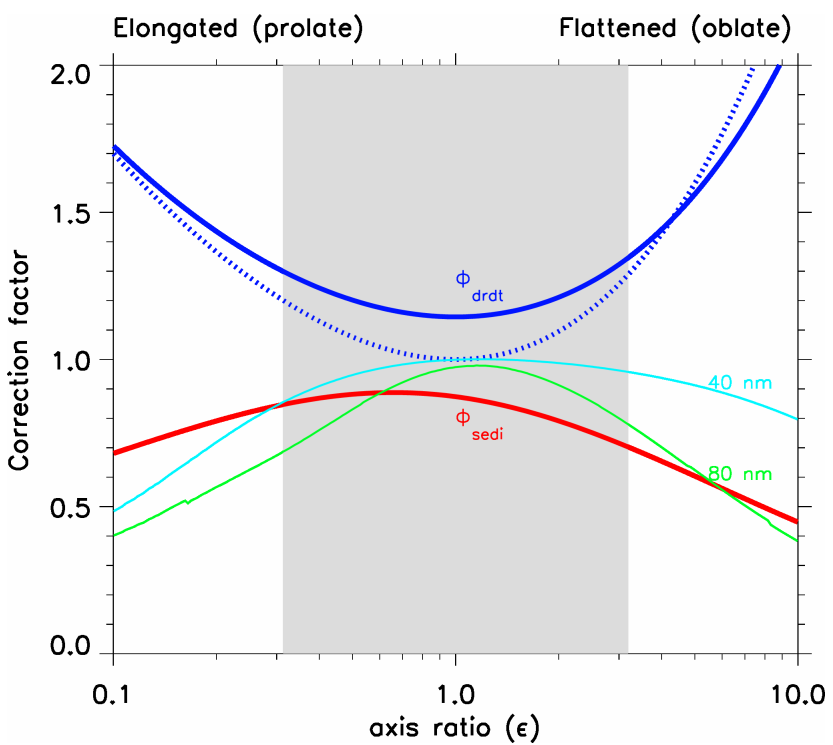

Figure 2. Correction factors $\Phi_{\mathrm{d} r \mathrm{~d} t}$ for particle growth and $\Phi_{\text {sedi }}$ for sedimentation of cylinders relative to growth and sedimentation of spheres. The dotted blue line shows $\Phi_{\mathrm{d} r \mathrm{~d} t}$ for spheroid particle shape. Thin lines show the backscatter signal $(532 \mathrm{~nm})$ ratio of same-volume cylinders to spheres for equivalent radii of 40 and $80 \mathrm{~nm}$. Shading indicates the $\varepsilon$ limits of moderately elongated or flattened particles consistent with satellite measurements (Hervig and Gordley, 2010).

For typical particle sizes in NLCs $(<100 \mathrm{~nm})$, cylindrical ice backscatters light at $532 \mathrm{~nm}$ less efficiently than spherical particles with the same equivalent radius. For needles with $\varepsilon=0.1$ and large discs with $\varepsilon=10$, the backscatter signal is less than half compared to spheres of the same volume (see Fig. 2). However, this does not necessarily imply that noctilucent clouds consisting of non-spherical particles are dimmer, as the growth and sedimentation mechanisms are affected by particle shape.

In summary, modeling of non-spherical particles is implemented by adjusting growth rate (Eq. 1), fall speed (Eq. 3), and the Mie scatter coefficient using the T-matrix method.

\subsection{Color ratios}

Optical methods for determining particle properties typically involve analyzing scatter or extinction signals at several wavelengths or scattering angles (e.g., von Cossart et al., 1999; Hervig et al., 2009a; McClintock et al., 2009). Since the Arctic Lidar Observatory for Middle Atmosphere Research (ALOMAR) Rayleigh/Mie/Raman (RMR) lidar uses $1064 \mathrm{~nm}$ (IR) and $355 \mathrm{~nm}$ (UV) in addition to the visible $532 \mathrm{~nm}$ (Vis) wavelength (Baumgarten et al., 2010), backscatter coefficients for these wavelengths are implemented in MIMAS as well. Color ratios (CRs) are defined as the relative scattering intensity of two wavelengths within the same sample volume. Unlike the backscatter signal $(\beta)$ at
NLC signal color ratios

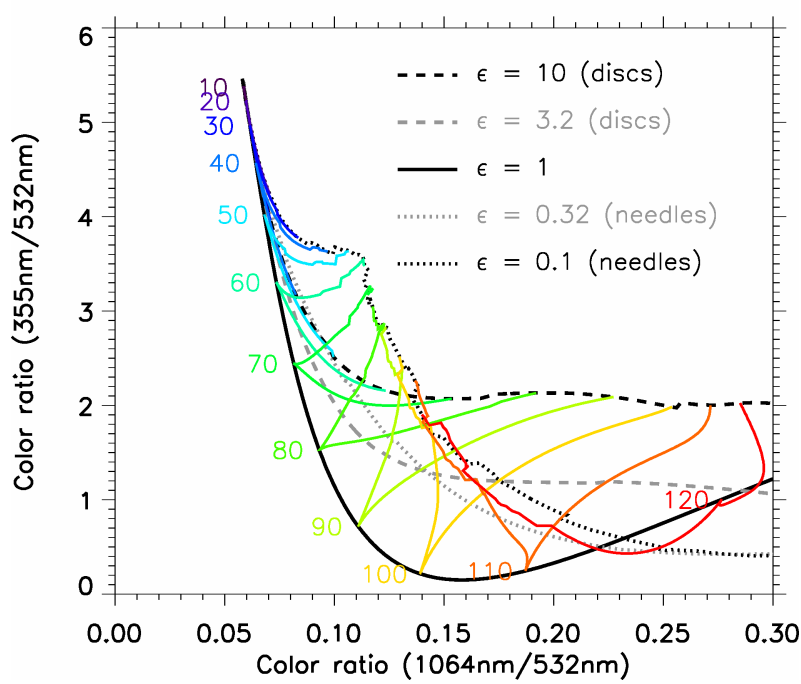

Figure 3. Combinations of color ratios UV / Vis (355 / $532 \mathrm{~nm})$ and IR / Vis (1064 / $532 \mathrm{~nm})$ for different particle equivalent radii $r$ and axis ratios $\varepsilon$, as calculated from Mie theory for cylindrical particles. Colored solid lines show constant $r$ with variable $\varepsilon$; the equivalent radius in nanometers is shown by the numbers near the $\varepsilon=1$ position (the line inflection is caused by very small $\frac{\mathrm{d} \beta}{\mathrm{d} \varepsilon}$ at $\varepsilon=1$ ). Line oscillations, mainly along the $\varepsilon<1$ branch, stem from rounding errors in the Mie scattering tables. The grey scale lines (dotted/dashed) show variable $r$ for fixed axis ratios, including cylinders with $\varepsilon=1$ for the solid black line.

a single wavelength, a color ratio is determined entirely by the size and shape of the particles within the sample volume, but not by their number density.

The ALOMAR RMR lidar uses $532 \mathrm{~nm}$ as a reference signal. From three wavelengths, two independent color ratios can be derived: $\mathrm{UV} / \mathrm{Vis}=\frac{\beta_{355 \mathrm{~nm}}(r)}{\beta_{532 \mathrm{~nm}}(r)}$ and $\mathrm{IR} / \mathrm{Vis}=\frac{\beta_{1064 \mathrm{~mm}}(r)}{\beta_{532 \mathrm{~mm}}(r)}$. Figure 3 shows the values of both color ratios for randomly oriented cylinders, as a function of volume-equivalent radius $r$ and axis ratio $\varepsilon$ in the parameter range $r<120 \mathrm{~nm}$ and $0.1<\varepsilon<10$. A uniform distribution of axial ratios $(0.1<\varepsilon<10)$ is used for the size retrievals as described in Baumgarten et al. (2007).

The color ratios of small particles asymptotically approach the limit of Rayleigh scattering. Since the refractive index of ice depends little on wavelength in the region of 355 to $1064 \mathrm{~nm}$ (e.g Hale and Querry, 1973), the following approximation can be used: $\beta_{\lambda}(r) \propto r^{2}\left(\frac{r}{\lambda}\right)^{4}$ if $r \ll \lambda$, and thus $\frac{\beta_{355 \mathrm{~nm}}(r)}{\beta_{532 \mathrm{~nm}}(r)} \longrightarrow\left(\frac{532 \mathrm{~nm}}{355 \mathrm{~nm}}\right)^{4} \approx 5$. Likewise for the IR / Vis ratio, $\frac{\beta_{1064 \mathrm{~nm}}(r)}{\beta_{532 \mathrm{~nm}}(r)}$ converges to a value of about 0.06 . This convergence is visible in Fig. 3 by the CR combinations of monodisperse distributions, where the lines for different axis ratios all intersect for small radii. Since small particles $(r<15 \mathrm{~nm})$ are difficult to detect by lidar, having similar color ratios makes it even more difficult to determine their size by optical meth- 
ods. This makes analyzing particle shape using color ratios much more feasible for large particles, which primarily occur in bright ice clouds.

Also shown in Fig. 3, the UV / Vis ratio is highest for small particles, while at larger sizes it decreases rapidly up to a minimum at $r \approx 100 \mathrm{~nm}$ (first UV resonance). In the size range 50 to $80 \mathrm{~nm}$, the UV/Vis ratio also generally decreases with increasing $\varepsilon$. The UV / Vis ratio of needles $(\varepsilon<1)$ is lower than for $\varepsilon=1$ particles with radii up to about $55 \mathrm{~nm}$, higher for larger particles. For disc-shaped particles $(\varepsilon=10)$, UV / Vis is smaller compared to $\varepsilon=1$ for $r<75 \mathrm{~nm}$ and larger for particles $>75 \mathrm{~nm}$. The IR / Vis ratio shows a continuous rise toward larger radii for $\varepsilon=1$ particles; this increase accelerates steeply around $100 \mathrm{~nm}$. For all particles smaller than $\approx 100 \mathrm{~nm}$, IR / Vis is always smaller for $\varepsilon=1$ cylinders than for elongated or flattened particles. Up to radii around $70 \mathrm{~nm}$, this increase is stronger for elongated $(\varepsilon<1)$ particles.

The color ratio curves in Fig. 3 also intersect: in many cases, there are multiple combinations of particle size and shape that fit a given (i.e., measured) pair of UV / Vis and IR / Vis ratios. However, no particle shape causes lower IR / Vis ratios for a given UV / Vis than spherical particles. This makes the area to the left of the solid black line a "forbidden area".

\section{Results}

\subsection{Comparison of modeled color ratios with observations}

For comparison with model results we use 30000 lidar measurements of color ratios observed in the period 1998 to 2014. In order to compare a wide range of NLC simulations with non-spherical shapes to these measurements, model simulations were conducted using background conditions from 5 days in mid-July of 2009, i.e., 120 time steps. July 2009 was selected for compatibility to previous simulations (Kiliani et al., 2013). For the model data, 5 latitude bands from 67 to $72^{\circ} \mathrm{N}$ were used, as well as 120 longitudinal bands (zonal model resolution is $3^{\circ}$ ).

The first simulation is the reference run with only spherical particle shape. Six model runs using very different distributions of non-spherical particles are also used, namely all shapes from highly elongated to very flat $(0.1<\varepsilon<$ $10)$, moderately flat $(1.1<\varepsilon<3.2)$, moderately elongated $(0.32<\varepsilon<0.87)$, moderately elongated to flat $(0.32<\varepsilon<$ $3.2)$, and moderately elongated to very flat $(0.32<\varepsilon<5.6$ and $0.32<\varepsilon<10$ ). For all simulations with cylindrical particles, the initial particle shape is distributed uniformly in $\log \varepsilon$; for instance the simulation with $(0.1<\varepsilon<10)$ includes the same number of particles with $(0.1<\varepsilon<0.2)$ as $(0.75<\varepsilon<1.5)$ or $(3<\varepsilon<6)$. The shape distribution is discussed in more detail later, in Fig. 9. All simulations used exactly the same atmospheric conditions (e.g., temperature, $\mathrm{H}_{2} \mathrm{O}$, wind).

For all these model simulations, all model grid volumes in the latitude range around ALOMAR $\left(69^{\circ} \mathrm{N}\right)$ are evaluated, $\approx 1$ million in the peak backscatter range. Since large particles are easier to distinguish using CRs (Sect. 2.3), the lidar statistic used here is restricted to strong NLCs with $\beta_{532}>13 \times 10^{-10} \mathrm{~m}^{-1} \mathrm{sr}^{-1}$. An equivalent restriction is used for the modeled NLC, which also removes the need for explicit altitude filtering. From the resulting volumes containing a strong NLC $(\approx 100000$ per simulation), a distribution of modeled NLC color ratios is computed for each of the seven simulations.

Figure 4 shows modeled color ratios from one of these simulations. Color ratios observed by lidar also include measurement uncertainties, which have to be simulated for the modeled NLC in order to compare color ratios directly. This is done by applying a Gaussian smoothing filter to the modeled CRs, with a filter width determined by the lidar measurement uncertainties, i.e., 0.01 (IR / Vis) and 0.3 (UV / Vis), respectively. Figure 4 shows the effect of the smoothing filter on the modeled color ratios for a simulation containing both needle- and disc-shaped particles. The unedited (modeled) CR distribution for $\beta>13 \times 10^{-10} \mathrm{~m}^{-1} \mathrm{sr}^{-1}$ covers a relatively narrow strip within the parameter space of IR / Vis and UV / Vis combinations, which roughly follows the line for spherical particles. For large particles (low UV / Vis) it diverges from the spherical particle line and becomes broader. As discussed in Sect. 2.3, the true CR distribution is sharply delimited by the spherical particle line: for ice particles smaller than $\approx 100 \mathrm{~nm}$, this line constitutes a minimum IR / Vis ratio using standard Mie theory.

After applying the smoothing filter, the distribution becomes broader and resembles a drop-like shape, lying partially in the forbidden area to the left of the spherical particle curve. Because of the wedge shape of the true distribution, the probability density maximum (innermost, red isolines) is also shifted toward lower UV / Vis values.

In Fig. 5 we compare modeled color ratios for strong NLCs in the spherical particle reference simulation to the lidar observations. The modeled distribution is drop-shaped like the one in Fig. 4, but with a steeper incline and aligned along the black curve for spherical particles.

The ALOMAR-measured color ratios also form a drop shape, but both its alignment and the position of its mode (the probability density distribution maximum) differ considerably from the spherical model simulation. In particular, the simulated mode in UV / Vis is higher (by 0.9) and the one in IR / Vis is lower (by 0.015) compared to the lidar. Also, the measured CRs include a long tail with IR / Vis ratios reaching up to 0.24 , which is not adequately reproduced by the spherical particle simulation (max IR / Vis: 0.13). In conclusion, using spherical ice particles produces color ratios which are not compatible with observations. 


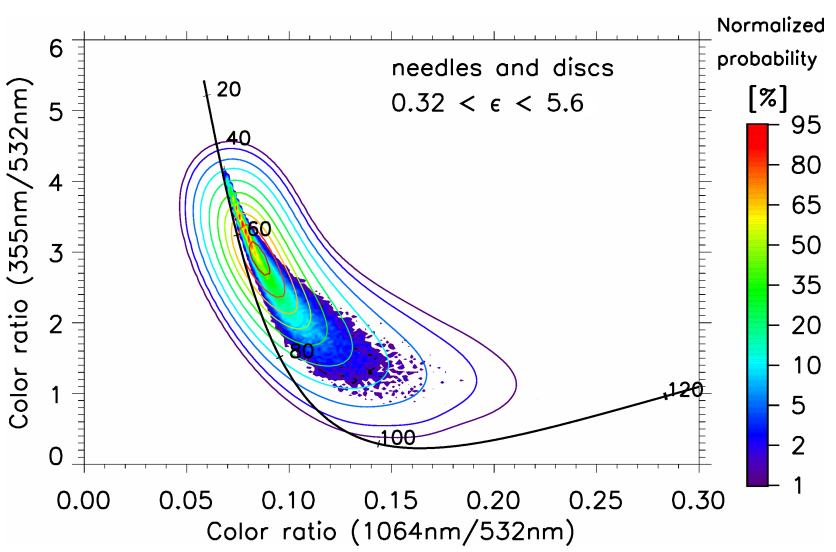

Figure 4. Filled contours: modeled distribution of color ratios for one NLC simulation with cylindrical particles, normalized to $100 \%$ for the most common CR combination. Contour lines: same distribution after applying a Gaussian smoothing filter determined by the uncertainty of lidar-measured color ratios. Black line: color ratios for spherical particles; numbers show CRs for particular spherical particle radii. These are very similar but not identical to the $\varepsilon=1$ cylinders in Fig. 3.

Figure 6 shows an extensive comparison of color ratios between the lidar data and six different cylindrical particle simulations, in the following referred to by their panel number in Fig. 6. Each panel includes a mean square deviation $\chi^{2}$ to quantify the degree of similarity between simulation and measurements:

$\chi^{2}=\frac{1}{n} \sum_{i=1}^{n}\left(X_{i}-\hat{X}_{i}\right)^{2}$,

where $X_{i}$ is the probability density of the modeled color ratios and $\hat{X}_{i}$ that of the lidar-measured CRs in a given CR bin. Lower values of $\chi^{2}$ indicate a better agreement.

The first simulation (a) represents a wide range of highly non-spherical cylinders $(0.1<\varepsilon<10)$. While this reproduces the measured distribution's tail adequately, the mode of the distribution is shifted towards higher IR / Vis to such an extent that $\chi^{2}$ is even larger than for the spherical run. Figure 3 shows that highly elongated particles around $60 \mathrm{~nm}$ leave a characteristic signature in color ratios, in the form of strongly increased IR / Vis compared to spherical particles, accompanied by relatively high UV / Vis values. The position of the lidar-measured CR mode thus counter-indicates the presence of such strongly needle-shaped ice particles to any great extent.

In the second model run (b), both disc- and needle-shaped particles are limited to moderate axis ratios $(0.32<\varepsilon<3.2)$. The comparison to the lidar data is much better with very similar distribution modes. The distribution tail is not reproduced very accurately, although considerably better than for the spherical reference run. This shape distribution is also consistent with satellite measurements, with the SOFIE

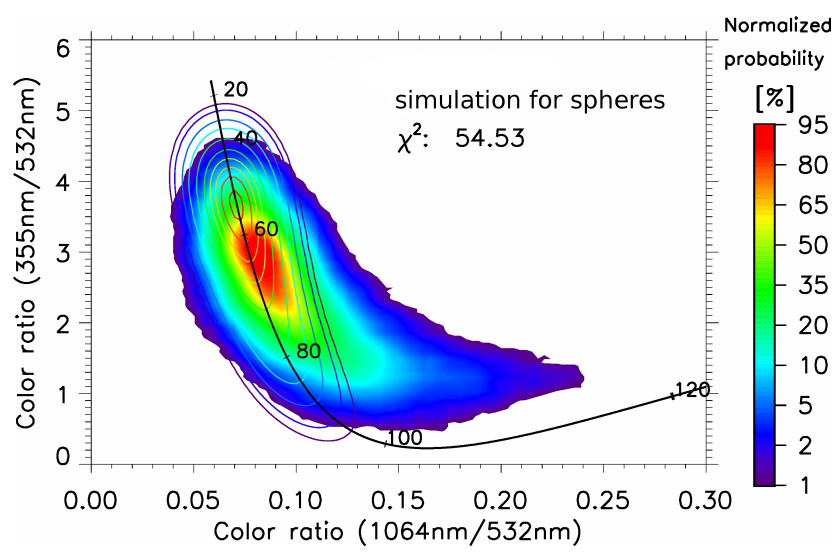

Figure 5. Filled contours: measured multi-year statistic of color ratio distribution observed by ALOMAR RMR lidar for strong NLCs $\left(\beta>13 \times 10^{-10} \mathrm{~m}^{-1} \mathrm{sr}^{-1}\right)$. Contour lines: modeled CR distribution for spherical particle simulation from 67 to $72^{\circ} \mathrm{N}$ with simulated measurement error. $\chi^{2}$ refers to the difference between model and measurements; see Fig. 6. All distributions are normalized to $100 \%$ for their respective probability density maximum.

instrument indicating mean axis ratios around 2. However, SOFIE is not able to distinguish between needle- and discshaped particles with the same ratio of longest to shortest axis. For this reason two additional simulations were conducted, one with only flattened particles $(1.1<\varepsilon<3.2)$ and another using only elongated cylinders $(0.32<\varepsilon<0.87)$.

Neither of the simulations (c) and (d) are an improvement compared to (b). In particular, the distribution tail is largely unchanged with only a slight improvement for (d). For the needle-shaped cylinders (d), the distribution mode is shifted away from the measurements, while the color ratios of the flattened particle simulation (c) are stretched out along the UV / Vis axis more than the lidar data set. The preliminary conclusion is that a combination of discs and needles is needed to accurately replicate both the mode and the tail of the lidar-measured distribution.

The remaining question is how to improve on (b) $(0.32<$ $\varepsilon<3.2$ ), primarily to get a better match for the distribution tail, like in (a). The two simulations (e) and (f) include more flattened ice particles while leaving the elongated part of the distribution unchanged compared to (b). The simulation (e) $(0.32<\varepsilon<5.6)$ shows even a slightly better match of the distribution modes than (b) while achieving a good approximation of the distribution tail. On the other hand, very highly flattened particles with $\varepsilon$ up to 10 (Fig. 6f) cause an exaggerated distribution tail and a shifting of the mode at the same time. The reason for the good match with (e) can be seen in Fig. 3: the relatively rare very large disc-shaped particles $>80 \mathrm{~nm}$ have much higher IR / Vis ratios compared to spheres or needles, which causes the tail and thus the drop shape of the color ratio distribution. 




Figure 6. Filled contours, all panels: lidar CR statistic as in Fig. 5. Contour lines: modeled color ratio distribution including simulated measurement errors for six different cylindrical particle shape distributions. All distributions are normalized to $100 \%$ for their respective maximum. $\chi^{2}$ values are calculated by averaging the squared deviations between model and observation (in $\%$ ) over the plot area, $0<\mathrm{UV} / \mathrm{Vis}<6$ and $0<\mathrm{IR} / \mathrm{Vis}<0.3$.

The analysis in Fig. 6 is qualitative to some extent because of its limited number of simulations, since the parameter space of possible particle shape distributions is much larger especially if non-uniform (e.g., Gaussian) distribution shapes are considered. A robust result is that cylindrical particles with axis ratios consistent with SOFIE (Hervig and Gordley, 2010) are also consistent with the ALOMAR lidar, that a mix of needle and disc shapes is required and that a slight emphasis on discs produces the best match.

\subsection{Effects on ice layers}

Since particle shape affects NLC microphysics in addition to optical cloud properties, the switch from spherical to cylindrical shape may affect the ice cloud morphology. Those effects are studied in this section, both in the average cloud morphology during mid-season and in the size distribution during a single, bright NLC event.

In Fig. 7 we compare observable parameters of simulated NLC layers at $69^{\circ} \mathrm{N}$ over a time period of 1 month (July of 2009). NLCs consisting of non-spherical particles are up to $50 \%$ brighter than those in the spherical particle simulation, resulting from both increased growth rates and reduced sedimentation compared to spheres (see Fig. 2). The brightness of the various non-spherical particle simulations are more similar: those favoring discs tend toward higher brightness than simulations with primarily needles, with differences in $\beta$ of up to $30 \%$. This disparity is caused by the stronger microphysical effects on growth and sedimentation for disc shapes as compared to needles (Fig. 2). The ice mass density of NLCs in simulations with cylindrical particles is also up to $\approx 30 \%$ higher than in the spherical particle run. As for the backscatter coefficient, the increase in ice mass is larger for simulations with disc-shaped cylinders than for needle shapes. The rather low values in average brightness and ice mass density result from the lack of a threshold. As minor changes in the mass density make some populations of clouds fall below the threshold in one simulation or the other, omitting a threshold gives more accurate comparisons for the different model runs. 


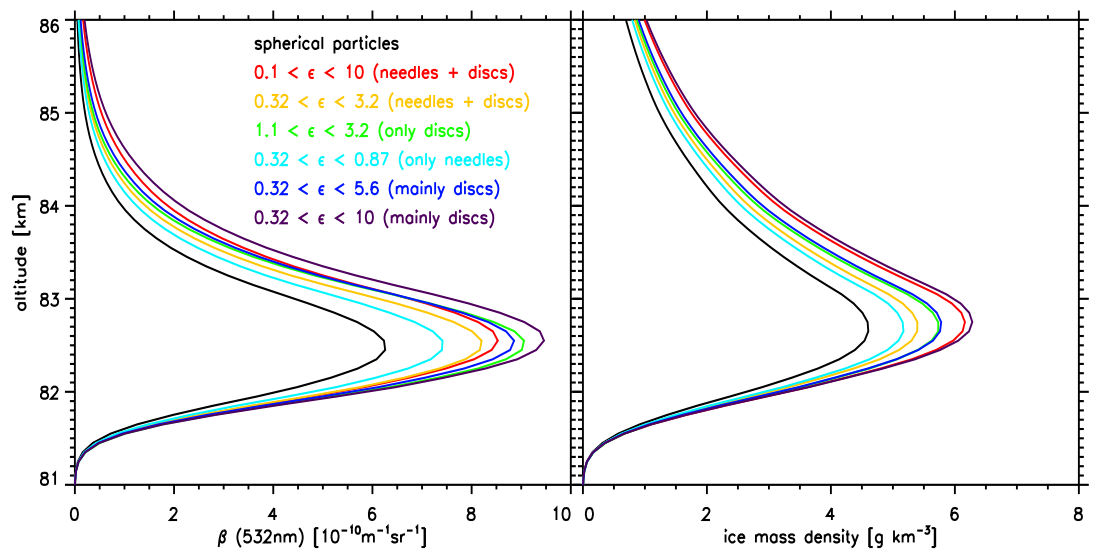

Figure 7. Average NLC parameters north of $60^{\circ} \mathrm{N}$ during July of 2009, from the reference simulation (spherical particles) and six sensitivity runs with different distributions of cylindrical particles. Left panel: backscatter coefficient $(532 \mathrm{~nm})$; right panel: ice mass density $\left(\mathrm{g} \mathrm{km}^{-3}\right)$. No threshold is used; i.e., zero values are included in the average.

Table 1. Mean values for MIMAS NLC brightness north of $60^{\circ} \mathrm{N}$ using different particle shape distributions.

\begin{tabular}{lrrr}
\hline Particle shape & $\begin{array}{r}\beta_{\max } \\
{\left[10^{-10} \mathrm{~m}^{-1} \mathrm{sr}^{-1}\right]}\end{array}$ & $\begin{array}{r}\beta_{\text {int }} \\
{\left[10^{-7} \mathrm{sr}^{-1}\right]}\end{array}$ & $\begin{array}{r}\Delta_{z} \\
{[\mathrm{~km}]}\end{array}$ \\
\hline Spherical & 6.26 & 9.58 & 1.53 \\
\hline Cylindrical & & & \\
$0.1<\varepsilon<10$ & 8.53 & 14.44 & 1.69 \\
$0.32<\varepsilon<3.2$ & 8.20 & 13.01 & 1.59 \\
$1.1<\varepsilon<3.2$ & 9.06 & 14.38 & 1.59 \\
$0.32<\varepsilon<0.87$ & 7.41 & 11.67 & 1.57 \\
$0.32<\varepsilon<5.6$ & 8.86 & 14.37 & 1.62 \\
$0.32<\varepsilon<10$ & 9.46 & 16.21 & 1.71 \\
\hline
\end{tabular}

The altitude of the NLC layer is much less affected by particle shape: all distributions have their brightness maximum within $100 \mathrm{~m}$ of each other, with the cylindrical particle simulations peaking at marginally higher altitude than the spherical reference run. Another parameter is the width of the mean ice layer, calculated as $\Delta_{z}=\frac{\beta_{\text {int }}}{\beta_{\max }}$, where $\beta_{\max }$ is the maximum brightness and $\beta_{\text {int }}$ the column-integrated brightness at $532 \mathrm{~nm}$ (e.g., Fiedler et al., 2009). It varies between $1.53 \mathrm{~km}$ for spherical particles and $1.71 \mathrm{~km}$ for the distribution favoring highly flattened particles; see Table 1 . Generally, the layer width increases with the axis ratios present in the shape distribution: the slower sedimentation and faster growth of non-spherical particles (especially disc-shaped) shifts the upper edge of the NLC region further up, while lower NLC edge and maximum altitude are less affected.

The altered particle shape also affects the particle size distribution and thus indirectly the backscatter signal in Fig. 7. In Fig. 8 we show the ice particle size and number density for a single strong NLC around $69^{\circ} \mathrm{N}$ at one time step (16 July 2009, 24:00 UT). In simulations with non-spherical

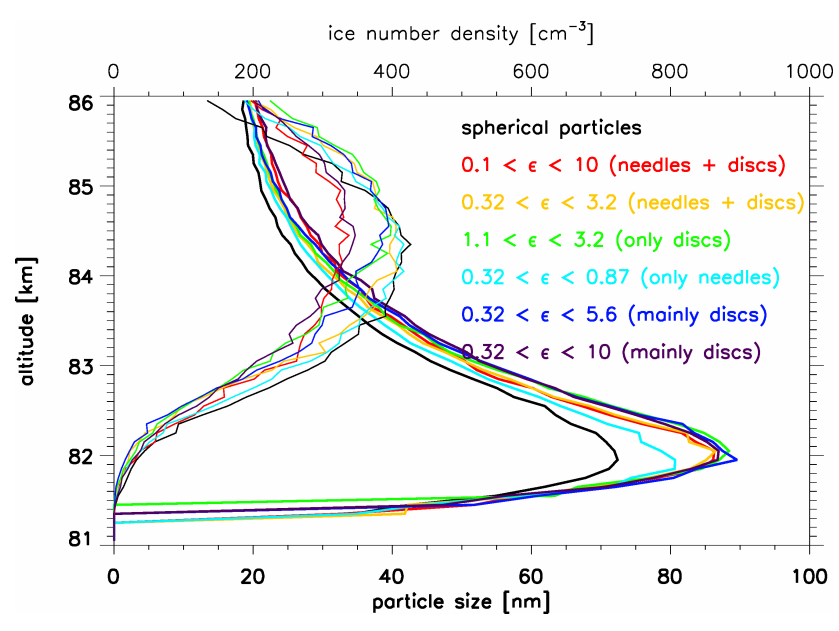

Figure 8. Simulated volume-equivalent radius and number density for a bright NLC event within $135-150^{\circ} \mathrm{E}, 67-72^{\circ} \mathrm{N}$ on 16 July 2009 at 24:00 UT, with different shape distributions. Only particles $>15 \mathrm{~nm}$ are included in the average of size and number density.

ice particles, these grow $\approx 5-10 \mathrm{~nm}$ larger on average, depending on altitude and the simulation in question. Among the non-spherical shapes, those with the highest aspect ratios form the largest particles, and flattened particles grow noticeably larger than elongated ones. The width of the particle size distribution is also larger for non-spherical shapes in general, with a strong increase in the presence of very large particles $(r>80 \mathrm{~nm}$, not shown in Fig. 8).

On the other hand, the number of ice particles in the main layer of this strong NLC is generally lower for the nonspherical shapes compared to the spherical shapes, by 20 $35 \%$. As with particle size, the largest differences in number density are seen for simulations including highly flattened particles. The increased size and decreased number density for non-spherical shape are linked to the availability of water 
Table 2. Microphysical parameters for the same strong NLC as in Fig. 8, compared between simulations with different particle shape distributions. The altitude of maximum brightness used for $n_{\max }, r_{\max }$ and $\sigma\left(r_{\max }\right)$ is $82-83 \mathrm{~km}$, and only particles larger than $15 \mathrm{~nm}$ are considered for all parameters.

\begin{tabular}{lrrrrrrr}
\hline Particle shape & $\begin{array}{r}n_{\max } \\
{\left[\mathrm{cm}^{-3}\right]}\end{array}$ & $\begin{array}{r}r_{\max } \\
{[\mathrm{nm}]}\end{array}$ & $\begin{array}{r}\sigma\left(r_{\max }\right) \\
{[\mathrm{nm}]}\end{array}$ & $\begin{array}{r}\bar{r} \\
{[\mathrm{~nm}]}\end{array}$ & $\begin{array}{r}\mathrm{IWC} \\
{\left[\mathrm{g} \mathrm{km}^{-2}\right]}\end{array}$ & $\begin{array}{r}\beta_{\max } \\
{\left[10^{-10} \mathrm{~m}^{-1} \mathrm{sr}^{-1}\right]}\end{array}$ & $\left.\begin{array}{r}\beta_{\text {int }} \\
{\left[10^{-7}\right.}\end{array} \mathrm{sr}^{-1}\right]$ \\
\hline Spherical & 151.3 & 57.6 & 11.5 & 31.7 & 266 & 63.7 & 67.3 \\
\hline Cylindrical & & & & & & & \\
$0.1<\varepsilon<10$ & 122.4 & 64.2 & 14.2 & 35.0 & 328 & 69.5 & 73.3 \\
$0.32<\varepsilon<3.2$ & 105.9 & 63.6 & 13.6 & 32.7 & 293 & 73.8 & 77.0 \\
$1.1<\varepsilon<3.2$ & 99.8 & 67.4 & 13.6 & 32.9 & 305 & 64.8 & 74.6 \\
$0.32<\varepsilon<0.87$ & 133.0 & 60.8 & 13.7 & 32.7 & 299 & 65.1 & 81.9 \\
$0.32<\varepsilon<5.6$ & 98.3 & 66.4 & 14.5 & 33.4 & 309 & 63.1 & 83.7 \\
$0.32<\varepsilon<10$ & 108.2 & 67.4 & 14.2 & 35.3 & 338 & & \\
\hline
\end{tabular}

vapor. For all simulations, the same initial $\mathrm{H}_{2} \mathrm{O}$ was used; this constrains the growth of NLC particles. The increased relative importance of turbulent transport compared to the (reduced) sedimentation rate results in a more effective differentiation of the ice layer into those particles growing visible $(r>20 \mathrm{~nm})$ and those staying at small size. For this reason, the larger particle size due to improved growth conditions for cylindrical ice is accompanied by a reduced number density in the NLC layer. Since the backscatter signal depends on particle size as $r^{5}$ to $r^{6}$, NLCs made of non-spherical particles are brighter than those with spherical particles in spite of the lower number density and reduced backscatter coefficient of single particles with the same equivalent radius.

Table 2 lists a number of additional microphysical parameters for the case of a strong cloud as shown in Fig. 8 for the (spherical) reference run and the various non-spherical particle simulations. The ice water content (IWC), defined as the column mass density of particles in the ice phase, shows a slight increase for cylindrical particle shapes, by 10$20 \%$. High axis ratio particles appear to increase IWC more than only slightly non-spherical shapes. The small increase supports the earlier statement that a limited supply of water vapor results in larger particles but reduced number density during strong growth conditions, i.e., when temperatures are low enough that a high fraction of the water vapor within the growth region is depleted by particle growth. IWC values for this event are generally high compared to satellite measurements (Hervig et al., 2009b; Hervig and Stevens, 2014), due to the choice of a very bright NLC. From Table 2 we see that the increase in backscatter signal for cylindrical particles is considerably weaker for this bright NLC example than for the statistic in Table 1. Only $\beta_{\text {int }}$ is consistently larger, similar to the results when analyzing all NLCs. As for the statistic in Table 1, flattened particles lead to brighter ice clouds, by 15-25\% compared to the spherical simulation.

Finally, Fig. 9 shows the development of ice particles within the six simulations with cylindrical ice particles. When counting all particles, the uniform initial distribution shapes (see Sect. 3.1) within the respective $\varepsilon$ limits are evident in all panels; minor deviations are due to statistical variability caused by the random-number generator. However, when counting only particles larger than specific radius thresholds, the resulting distributions are no longer uniform but constitute a U shape: strongly non-spherical particles are considerably more common than those with $\varepsilon$ close to 1 if only large particles are considered. For the simulation (a) with $(0.1<\varepsilon<10)$, highly flattened particles $(\varepsilon=10)$ are around $70 \%$ more common than $\varepsilon=1$ particles, if the radius threshold is set at $5 \mathrm{~nm} . \varepsilon=10$ is 3 times more common than $\varepsilon=1$ for particles with $r>10 \mathrm{~nm}$ and nearly 6 times more common for $r>20 \mathrm{~nm}$. This imbalance is smaller but still distinct for elongated or more moderately flattened cylinders. It also appears to be largest for size thresholds around $20 \mathrm{~nm}$, since the imbalance is slightly smaller for a threshold of $40 \mathrm{~nm}$ (visible NLC particles).

These differences in Fig. 9 are much larger than those between simulations in Figs. 7 and 8: with their larger surfaceto-volume ratio, strongly non-spherical particles outperform low $\varepsilon$ particles in growing to large size in a common volume. This is observed both for elongated and flattened high- $\varepsilon$ particles but is most pronounced for the flattened (disc-shaped) case. The prevalence of high axis ratios among large particles is most likely due to the increased growth rates, with the reduced fall speed contributing slightly at most. Otherwise we would expect the center of the U shape shifted to elongated particles, like for the correction factor $\Phi_{\text {sedi }}$; see Fig. 2. Shape inhomogeneities in the general particle distribution tend to get amplified within the NLC layer: Fig. 9 includes the average axis ratio $\langle\varepsilon\rangle$ for each radius threshold, calculated using $\frac{1}{\varepsilon}$ for $\varepsilon<1$. $\langle\varepsilon\rangle$ is shifted to higher values when only large $(r>20 \mathrm{~nm})$ particles are considered, by $\approx 0.1$ in simulations (b), (c), and (d) and by 1.2 and 1.4 in simulations (a) $(0.1<\varepsilon<10)$ and (f) $(0.32<\varepsilon<10)$, respectively. This helps to explain the large effects on the optical NLC properties in Sect. 3.1 seen for simulations including highly aspheric particles. For simulation (e) $(0.32<\varepsilon<5.6)$, where 


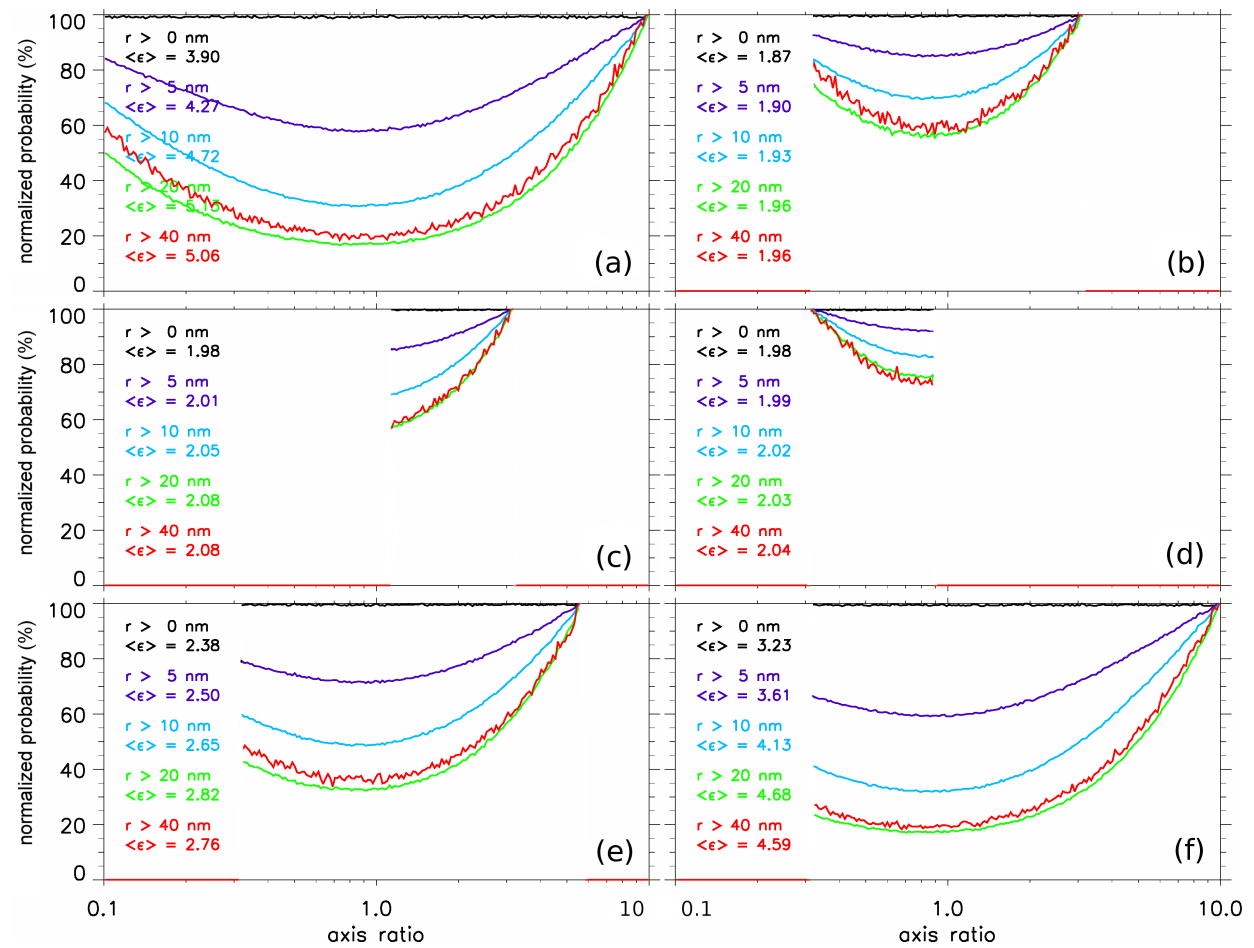

Figure 9. Shape distribution of simulations with non-spherical particles on 16 July 2009 at 24:00 UT; panels (a) to (f) are arranged analogous to Fig. 6. Black lines are the initial $(\log \varepsilon)$ uniform distributions including all ice particles; colored lines show shape distributions for particles larger than a given threshold. For each radius threshold the distributions are individually normalized to the most common particle shape, and mean $\varepsilon$ values $(\langle\varepsilon\rangle)$ are calculated using $\frac{1}{\varepsilon}$ for $\varepsilon<1$.

we find the best agreement to the ALOMAR lidar, the $\langle\varepsilon\rangle$ value of 2.8 for $r>20 \mathrm{~nm}$ is more suitable for comparing mean axis ratios to SOFIE satellite measurements than the lower value of 2.4 for the initial distribution since the backscatter signal is caused by large ice particles. Our analysis thus yields a somewhat higher estimate for mean axis ratio than the value of 2.0 by Hervig and Gordley (2010).

\section{Discussion and conclusions}

Size and shape of noctilucent cloud particles have long been an important topic in characterizing ice formation in the upper mesosphere region. While the particle size has been studied extensively, spherical shape is commonly assumed for mesospheric ice particles, especially in model studies. In this paper, several distributions of non-spherical ice particles are studied. To limit computational complexity, particles are assumed to be rotationally symmetric, in particular cylindrical shaped, and particle shape is further assumed to stay constant in time. This last assumption was chosen as a simple way to treat axis ratio effects on the microphysics. Uniform condensation on an elongated or flattened particle would continually decrease its axial ratio during growth. On the other hand the crystalline structure of ice or particle charge could counteract this to increase axial ratios. An implementation of all effects would require much more complex microphysics, which is beyond the scope of the paper.

The optical properties of these model ice particles are compared with measurements: while a lidar is capable of measuring shape information directly through depolarization, this is not done routinely (Baumgarten et al., 2002). Using the relative strength of the scatter signal in three different wavelengths (color ratios) gives a larger and more robust data set for comparison with models.

Rapp et al. (2007) found that color ratios from NLCs as measured by lidar do not match the model simulation well if it is assumed that particles are of spherical shape. For 11 color ratio measurements in 1998 by von Cossart et al. (1999) they found that needles with axial ratios of $1 / 5$ or plates with an axial ratio of 7 explain the observations.

With an extended data set of about 30000 color ratio measurements and improved modeling the agreement is much better for simulations with cylindrical particles, particularly if both elongated and flattened particles are included. The simulation most consistent with the lidar $(0.32<\varepsilon<5.6)$ includes $\approx 60 \%$ more discs $(\varepsilon>1.25)$ than needles $(\varepsilon<0.8)$, with a mean $\varepsilon$ of 2.8. From the good agreement of color ratios we infer that the ice clouds observed by lidar have a distribution of both particle size and shape very similar to the model run. 
The model simulations with cylindrical particles generally produce brighter ice layers, mainly because their shape slows sedimentation compared to spherical particles and thus enables them to grow to larger sizes. Mean NLC brightness $\left(\beta_{532 \mathrm{~nm}}\right)$ is also increased by up to $50 \%$; yet this affects weaker ice clouds more than strong NLCs: comparing various simulations for the case of a strong NLC, the upper end of the particle size distribution is shifted towards considerably larger particles for the non-spherical case. The mean size is $5-10 \mathrm{~nm}$ larger for the non-spherical particles, accompanied by a broadening of the size distribution. Since particle number densities for the non-spherical particle simulations are lower, the brightness increase is less than would be expected from particle size alone. Simulations using nonspherical particles also feature a modest increase in ice mass (IWC) of up to $30 \%$, reduced to $10-20 \%$ when considering only strong ice clouds.

Using cylindrical instead of spherical particle shape in NLC modeling makes the simulated optical cloud properties consistent with lidar and satellite observations. The effects on NLC microphysics are less pronounced: cylindrical NLC particles are slightly larger, but the corresponding increase in ice water content and cloud brightness is partly compensated for by reduced number densities.

To conclude, the effects of using non-spherical particles on optical NLC properties such as color ratios or scattering angles (for satellites) are considerable and important for comparing simulations to measurements.

Acknowledgements. This research was sponsored by the German Federal Ministry of Education and Research through the Role Of the Middle atmosphere In Climate (ROMIC) project Trends In the Middle Atmosphere (TIMA), grant number 01LG1210A. We gratefully acknowledge the European Centre for Medium-Range Weather Forecasts (ECMWF) for providing the ERA-40 reanalysis data used in the simulations.

Edited by: W. Ward

\section{References}

Bardeen, C. G., Toon, O. B., Jensen, E. J., Hervig, M. E., Randall, C. E., Benze, S., Marsh, D. R., and Merkel, A.: Numerical simulations of the three-dimensional distribution of polar mesospheric clouds and comparisons with Cloud Imaging and Particle Size (CIPS) experiment and the Solar Occultation For Ice Experiment (SOFIE) observations, J. Geophys. Res., 115, D10204, doi:10.1029/2009JD012451, 2010.

Baumgarten, G., Fricke, K. H., and von Cossart, G.: Investigation of the shape of noctilucent cloud particles by polarization lidar technique, Geophys. Res. Lett., 29, 8.1-8.4, doi:10.1029/2001GL013877, 2002.

Baumgarten, G., Fiedler, J., and von Cossart, G.: The size of noctilucent cloud particles above ALOMAR (69N,16E): Optical mod- eling and method description, Adv. Space Res., 40, 772-784, doi:10.1016/j.asr.2007.01.018, 2007.

Baumgarten, G., Fiedler, J., and Rapp, M.: On microphysical processes of noctilucent clouds (NLC): observations and modeling of mean and width of the particle size-distribution, Atmos. Chem. Phys., 10, 6661-6668, doi:10.5194/acp-10-6661-2010, 2010.

Berger, U.: Modeling of middle atmosphere dynamics with LIMA, J. Atmos. Sol.-Terr. Phy., 70, 1170-1200, doi:10.1016/j.jastp.2008.02.004, 2008.

Berger, U. and Lübken, F.-J.: Weather in mesospheric ice layers, Geophys. Res. Lett., 33, L04806, doi:10.1029/2005GL024841, 2006.

Berger, U. and Lübken, F.-J.: Trends in mesospheric ice layers in the Northern Hemisphere during 1961-2013, J. Geophys. Res.Atmos., 120, doi:10.1002/2015JD023355, online first, 2015.

Berger, U. and von Zahn, U.: Icy particles in the summer mesopause region: three-dimensional modeling of their environment and two-dimensional modeling of their transport, J. Geophys. Res., 107, 1366, doi:10.1029/2001JA000316, 2002.

Berger, U. and von Zahn, U.: Three-dimensional modeling of the trajectories of visible noctilucent cloud particles: an indication of particle nucleation well below the mesopause, J. Geophys. Res., 112, D16204, doi:10.1029/2006JD008106, 2007.

Eremenko, M. N., Zasetsky, S. V. P. A. Y., Karlsson, B., Rinsland, C. P., Llewellyn, E. J., and Sloan, J. J.: Shape and composition of PMC particles derived from satellite remote sensing measurements, Geophys. Res. Lett., 32, L16S06, doi:10.1029/2005GL023013, 2005.

Fiedler, J., Baumgarten, G., and Lübken, F.-J.: NLC observations during one solar cycle above ALOMAR, J. Atmos. Sol.-Terr. Phy., 71, 424-433, doi:10.1016/j.jastp.2008.11.010, 2009.

Gadsden, M.: A note on the orientation and size of noctilucent cloud particles, Tellus B, 35, 73-75, doi:10.1111/j.16000889.1983.tb00009.x, 1983.

Hale, G. M. and Querry, M. R.: Optical constants of water in the 200-nm to 200-micrometer wavelength region, Appl. Optics, 12, 555-563, doi:10.1364/AO.12.000555, 1973.

Hervig, M. E. and Gordley, L. L.: Temperature, shape, and phase of mesospheric ice from Solar Occultation for Ice Experiment observations, J. Geophys. Res., 115, D15208, doi:10.1029/2010JD013918, 2010.

Hervig, M. E. and Stevens, M. H.: Interpreting the 35 year SBUV PMC record with SOFIE observations, J. Geophys. Res., 119, 12689-12705, doi:10.1002/2014JD021923, 2014.

Hervig, M. E., Gordley, L. L., Stevens, M. H., Russell III, J. M., Bailey, S. M., and Baumgarten, G.: Interpretation of SOFIE PMC measurements: cloud identification and derivation of mass density, particle shape, and particle size, J. Atmos. Sol.-Terr. Phy., 71, 316-330, doi:10.1016/j.jastp.2008.07.009, 2009a.

Hervig, M. E., Stevens, M. H., Gordley, L. L., Deaver, L. E., Russell, J. M., and Bailey, S. M.: Relationships between polar mesospheric clouds, temperature, and water vapor from Solar Occultation for Ice Experiment (SOFIE) observations, J. Geophys. Res. 114, D20203, doi:10.1029/2009JD012302, 2009b.

Hobbs, P. V., Chang, S., and Locatelli, J. D.: The dimensions and aggregation of ice crystals in natural clouds, J. Geophys. Res., 79, 2199-2206, doi:10.1029/JC079i015p02199, 1974. 
Holton, J. R.: The influence of gravity wave breaking on the general circulation of the middle atmosphere, J. Atmos. Sci., 40, 2497-2507, doi:10.1175/15200469(1983)040<2497:TIOGWB>2.0.CO;2, 1983.

Kiliani, J.: 3-D Modeling of Noctilucent Cloud Evolution and Relationship to the Ambient Atmosphere, $\mathrm{PhD}$ thesis, Universität Rostock, Rostock, Germany, 2014.

Kiliani, J., Baumgarten, G., Lübken, F.-J., Berger, U., and Hoffmann, P.: Temporal and spatial characteristics of the formation of strong noctilucent clouds, J. Atmos. Sol.-Terr. Phy., 104, 151166, doi:10.1016/j.jastp.2013.01.005, 2013.

Lübken, F., Berger, U., and Baumgarten, G.: Stratospheric and solar cycle effects on long-term variability of mesospheric ice clouds, J. Geophys. Res., 114, D00I06, doi:10.1029/2009JD012377, 2009.

Lübken, F.-J., Berger, U., Kiliani, J., Baumgarten, G., and Fiedler, J.: Solar variability and trend effects in mesospheric ice layers, in: Climate and Weather of the Sun-Earth System (CAWSES), edited by: Lübken, F.-J., Springer, Dordrecht, 317338, doi:10.1007/978-94-007-4348-9_18, 2013.

McClintock, W. E., Rusch, D. W., Thomas, G. E., Merkel, A. W., Lankton, M. R., Drake, V. A., Bailey, S. M., and Russell, III, J. M.: The cloud imaging and particle size experiment on the Aeronomy of Ice in the mesosphere mission: instrument concept, design, calibration, and on-orbit performance, J. Atmos. Sol.Terr. Phy., 71, 340-355, doi:10.1016/j.jastp.2008.10.011, 2009.

Megner, L.: Minimal impact of condensation nuclei characteristics on observable Mesospheric ice properties, J. Atmos. Solar-Terr. Phy., 73, 2184-2191, doi:10.1016/j.jastp.2010.08.006, 2011.

Merkel, A. W., Marsh, D. R., Gettelman, A., and Jensen, E. J.: On the relationship of polar mesospheric cloud ice water content, particle radius and mesospheric temperature and its use in multi-dimensional models, Atmos. Chem. Phys., 9, 8889-8901, doi:10.5194/acp-9-8889-2009, 2009.
Mishchenko, M. I. and Travis, L. D.: Capabilities and limitations of a current FORTRAN implementation of the T-matrix method for randomly oriented, rotationally symmetric scatterers, J. Quant. Spectrosc. Ra., 60, 309-324, doi:10.1016/S00224073(98)00008-9, 1998.

Murray, B. J., Malkin, T. L., and Salzmann, C. G.: The crystal structure of ice under mesospheric conditions, J. Atmos. Sol.-Terr. Phy., 127, 78-82, doi:10.1016/j.jastp.2014.12.005, 2015.

Pruppacher, H. R. and Klett, J. D.: Microphysics of Clouds and Precipitation, Kluwer Academic Publishers, Dordrecht, 1997.

Rapp, M., Thomas, G. E., and Baumgarten, G.: Spectral properties of mesospheric ice clouds: evidence for non-spherical particles, Geophys. Res. Lett., 112, D03211, doi:10.1029/2006JD007322, 2007.

Turco, R. P., Toon, O. B., Whitten, R. C., Keesee, R. G., and Hollenbach, D.: Noctilucent clouds - simulation studies of their genesis, properties and global influences, Planet. Space Sci., 30, 1147 1181, doi:10.1016/0032-0633(82)90126-X, 1982.

von Cossart, G., Fiedler, J., and von Zahn, U.: Size distributions of NLC particles as determined from 3-colour observations of NLC by ground-based Lidar, Geophys. Res. Lett., 26, 15131516, doi:10.1029/1999GL900226, 1999.

von Zahn, U. and Berger, U.: Persistent ice cloud in the midsummer upper mesosphere at high latitudes: three-dimensional modeling and cloud interactions with ambient water vapor, J. Geophys. Res., 108, 8451, doi:10.1029/2002JD002409, 2003. 